Proc. XII Int. School on Theoretical Physics — Symmetry and Structural Properties of Condensed Matter

\title{
The Dependence of the Proton Conductivity on Concentration for Networks with Different Symmetry of Molecules
}

\author{
T. MASŁOWSKI ${ }^{a * *}$ AND K.K. DUDEK ${ }^{b}$ \\ ${ }^{a}$ The Faculty of Mathematics and Applied Physics, Rzeszów University of Technology, \\ al. Powstańców Warszawy 6, 35-959 Rzeszów, Poland \\ ${ }^{b}$ Metamaterials Unit, Faculty of Science, University of Malta, Msida MSD 2080, Malta
}

\begin{abstract}
The one-dimensional numerical model of the proton conductivity in anhydrous solids is used to obtain the conductivity for different values of proton concentration. The model is constructed as a chain of rods or triangles, depending on the symmetry of molecules, linked by hydrogen bonds. An evolution of such system governed by the Grotthuss mechanism is described by means of the kinetic Monte Carlo method. It is shown that in the case of a system with molecules represented by triangles, a decrease in the conductivity corresponds to a significantly broader range of concentrations than is the case for a system constructed by means of rods. This result may prove to be of a potential importance in the case of possible electronic applications. Moreover, for the discussed systems, a strong dependence on the defect formation energies is demonstrated.
\end{abstract}

DOI: 10.12693/APhysPolA.132.129

PACS/topics: 66.30.Dn, 66.30.jp, 82.20.Wt

\section{Introduction}

Over the years, proton diffusion has been intensively investigated due to its potential use in applications such as fuel cells (PEFCs) [1], batteries [2] and supercapacitors [3] which in turn may be used in a wide variety of electronic devices (e.g. smart phones etc.). Even though this phenomenon might be observed for a vast number of systems, a lot of attention was devoted to solids, as these systems (as opposed to hydrous proton conductors) do not undergo a rapid decrease of proton diffusion above the boiling temperature of water.

One of the main mechanisms governing proton diffusion in solids is the Grotthuss mechanism, a two-stage mechanism of proton migration consisting of thermally induced reorientations of molecules and proton hoppings between minima of the hydrogen-bond (H-bond) potential [4]. This kind of diffusion mechanism is presented in solid systems such as solid acids, anhydrous heterocyclic based polymers and perovskites, which systems may correspond to very large values of proton conductivity. It is also worth noting that for a broad class of compounds the proton diffusion is realized along one-dimensional path (e.g. $\mathrm{CsHSO}_{4}$ ), which allows to simulate a proton diffusion solely by means of a one-dimensional chain of molecules [5].

\section{Model}

In this work, it is assumed that a one-dimensional path along which the proton diffusion is observed, corresponds to a chain of molecules which may be represented by appropriate polygons (it is determined by their symmetry)

*corresponding author; e-mail: tmaslowski@prz.edu.pl having their vertices occupied either by protons or vacancies. More specifically, two particular geometries are going to be discussed, namely rods and equilateral triangles, corresponding to molecules of heterocyclic based polymers and solid acids with tetrahedral molecules respectively. One may also note that in the case of the systems consisting of perovskites, the octahedral molecules could be represented by squares.

The reorientation process governed by the Grotthuss mechanism consists both of rotation of molecules and hopping of protons between the adjacent molecules. In the case of an investigated system consisting of rigid bars (see Fig. 1a), the rotation process manifests itself by the flipping of individual bars by $180^{\circ}$. On the other hand, in the case of the system constructed by means of triangles (see Fig. 1b), the rotation corresponds to a discrete value of $\pm 120^{\circ}$. Such representation of the rotation of the tetrahedral molecules stems from the fact that on each molecule one of the vertices remains fixed with respect to the axis of rotation passing through the molecule.

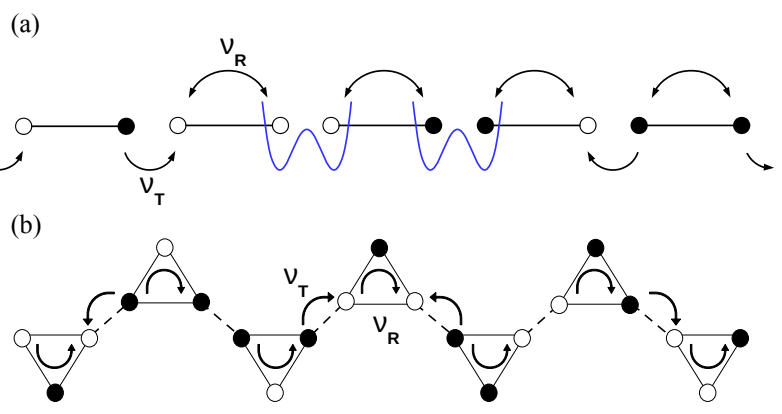

Fig. 1. (a) The chain of rods with marked positions of protons (solid dots) and vacancies (empty dots). Arrows denote allowed movements, while wave lines stand for hydrogen bonds (two exemplary positions). (b) The same as (a) with triangles instead of rods. 
The second allowed type of movement in the chain concerns proton hoppings between the two adjacent molecules, which process represents proton tunneling in the hydrogen bond.

Rotations and hoppings, are characterized by their characteristic frequencies denoted accordingly by $\nu_{R}$ and $\nu_{T}$. These quantities may be obtained from the experiment or determined from theory. In the theoretical approach reliable results may be obtained even when using the Bell theory [6] - the simplest way of taking quantum tunneling into account for activation processes. In this work, the values of $\nu_{R}$ and $\nu_{T}$ are representative for solid acids at room temperature.

At this point, it is important to highlight the fact that it is energetically unfavorable for two protons or two vacancies to occupy both minima of the same H-bond. Such configurations are called the Bjerrum D and L defects, respectively (Fig. 2). The energy of such configuration is of the order of magnitude of $V_{C} \approx 0.4 \mathrm{eV}$ [7].

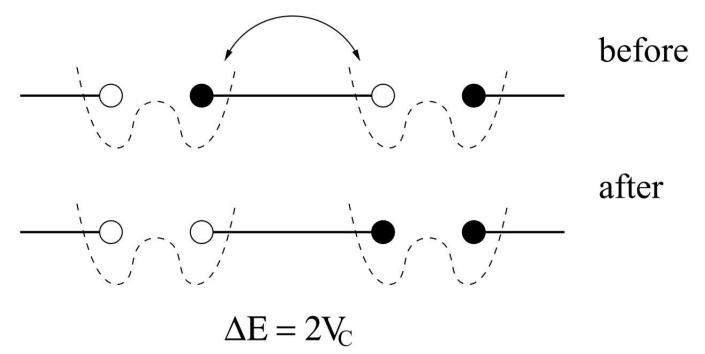

Fig. 2. An example of configuration yielding to the Bjerrum defect in the case of the rods model. After the rotation both, D and L, defects appear. Thus the energy difference between initial and final configuration is $2 V_{C}$.

In the case of the considered model, vertices of the respective molecules within the system may be occupied either by protons or vacancies. A particular number of protons within a given system may be established by means of the proton concentration $c$. This quantity is defined as the ratio of protons in the system to the number of all vertices. At this point, it is important to note that in real materials, the value of $c$ is fixed and depends on the symmetry, properties of bonds, impurities and distortion.

In this work, in order to investigate a time evolution of the discussed system, the kinetic Monte Carlo method was used. In this approach, the proton current is calculated by counting protons crossing a specified position in the chain [5]. In order to observe a flow of protons the electric field was applied to the system. Because we are interested in dc current it is necessary to impose periodic boundary conditions to avoid polarization of the sample.

\section{Conductivity}

In this work, a proton diffusion was investigated for two different systems in which molecules are represented by rods and triangles. In both of these cases, the results concerning proton diffusion plotted with respect to $c$ were generated for a discrete set of values of the Bjerrum defect formation energy, $V_{C}$ (see Figs. 3 and 4). One should also note that in each of the considered cases, values of $c$ are ranging between 0 and 1 with a discrete step $\Delta c$. Moreover, all of the points in the provided graphs correspond to separate kinetic Monte Carlo simulations.

Based on Figs. 3 and 4, one may note that in both cases of the discussed types of systems, the conductivity curves are symmetric with respect to $c=1 / 2$. This may be considered to be a manifestation of the protonvacancy symmetry in the investigated models. Another similarity which may be easily observed in both sets of results is a strong dependence of the proton diffusion on $V_{C}$.

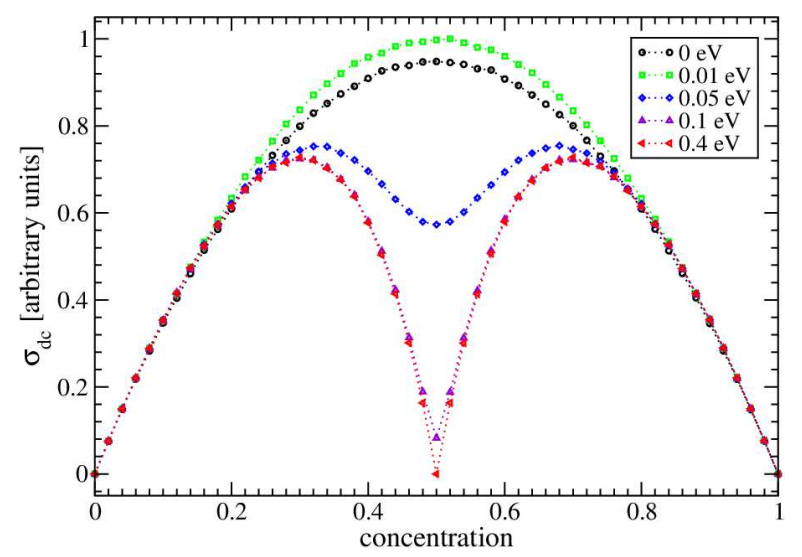

Fig. 3. The conductivity as a function of proton concentration for the chain of rods. Values in the legend refer to different energies of the Bjerrum defects.

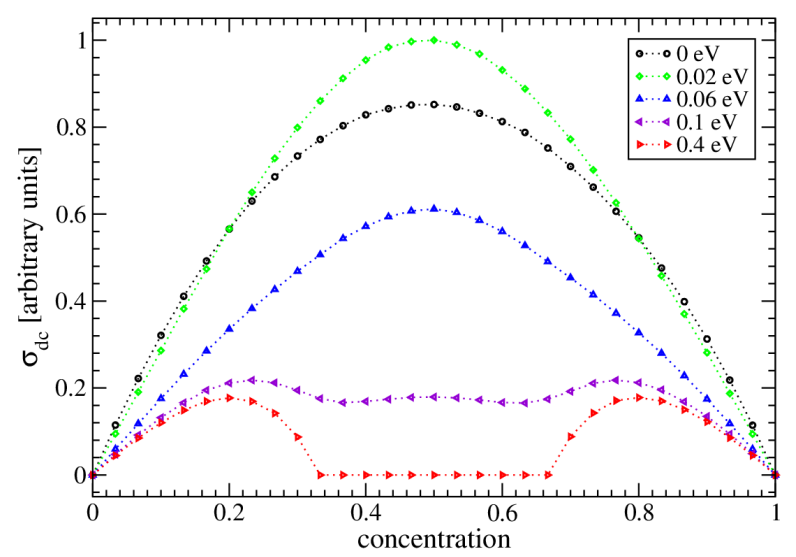

Fig. 4. The conductivity for the chain of triangles. The rest as in Fig. 3.

In the case of the system consisting of rods, the dependence of the proton diffusion on $V_{C}$ may be understood as explained in [5]. The absence of $V_{C}$ allows for a double occupancy of H-bond minima which effectively means that all proton positions are equivalent, i.e. the grouping of points in pairs on rods is only formal. One can also note that for $V_{C}=0 \mathrm{eV}$, the conductivity has a maximum at $c=1 / 2$. This particular result may be explained upon assuming that the diffusion is realized by propaga- 
tion of defects, as in such the case, the probability of a defect appearance is the highest for $c=1 / 2$. Furthermore, according to Fig. 3, for relatively small values of $V_{c}\left(V_{c} \neq 0\right)$, conductivity is increasing in comparison to the case corresponding to $V_{C}=0 \mathrm{eV}$. This means that a small formation energy of defects increases the diffusion. One may also note that a further growth of $V_{C}$ does not affect results corresponding to relatively small values of $c$ or $1-c$. It seems that only results corresponding to $c$ having values in the vicinity of $1 / 2$ are significantly affected by an increase in $V_{C}$. For large enough values of $V_{C}\left(V_{C} \approx 0.4 \mathrm{eV}\right)$ the proton conductivity decreases significantly in the vicinity of $c=1 / 2$. This particular behavior may also be explained by means of the concept of a symmetry [5]. In this case (one proton per rod), it is difficult to generate defects necessary for the effective diffusion, which leads to the aforementioned decrease in the proton conductivity.

In the case of a model consisting of triangles, for relatively small values of $V_{C}$, the conductivity of the system exhibits a similar behavior to the one observed for the rod model. On the other hand, for relatively large values of $V_{c}\left(V_{c} \approx 0.1 \mathrm{eV}\right)$ a more complex behavior may be observed. In those cases, the proton conductivity has three maxima corresponding to values of $c$ at around $0.25,1 / 2$ and 0.75 , separated by two minima near $1 / 3$ and $2 / 3$ (see Fig. 4). Further growth of $V_{C}$ leads to one deep wide minimum extending from $c=1 / 3$ to $2 / 3$. The symmetry arguments, as it was in the case of rods, may be applied to points $c=1 / 3$ and $2 / 3$ (one proton or one vacancy per triangle, respectively). However, for the rest of the interval between these points the blocking of proton diffusion seems to have a dynamical nature. For $c<1 / 3$ and $c>2 / 3$ there is always a triangle without protons/vacancies and thus a place for a defect to occur. For $1 / 3<c<2 / 3$ all triangles have an average of at least one proton or vacancy.

A proton must have a free vertex to hop further, but this is difficult to get when there is already another proton on the neighbor triangle.

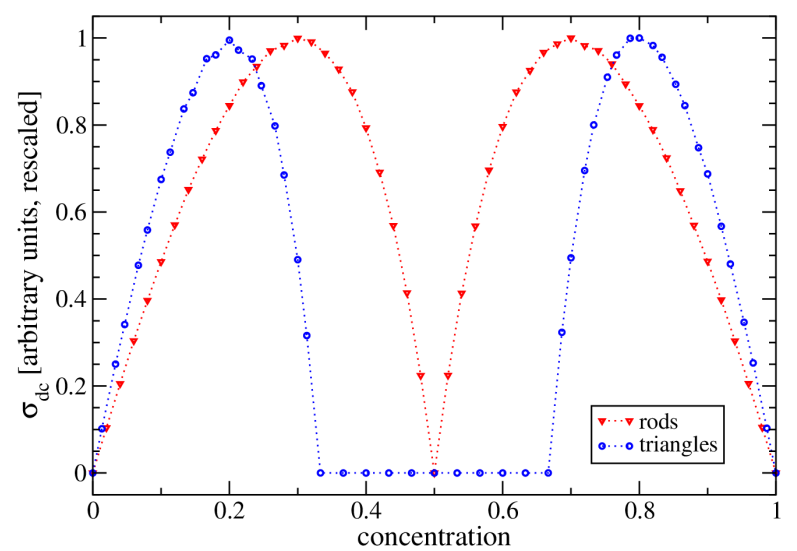

Fig. 5. The comparison of conductivities for rods and triangles (both independently scaled to 1). The Bjerrum defect energy equal to $0.4 \mathrm{eV}$.
In both cases considered here, physically reasonable values of $V_{C}$ are in the region recognized as large $V_{C}$. Then, the possibility of changing the proton concentration from $c \approx 1 / 2$ would results in significant rise of the conductivity (see Fig. 5), even of several orders of magnitude. The increase of the conductivity could also be obtained by lowering $V_{C}$. An experimental realization of such changes is still an open question.

\section{Conclusions}

Through the kinetic Monte Carlo algorithm, the proton diffusion was investigated in the case of two different systems corresponding to heterocyclic based polymers (constructed by means of rods) and solid acids (represented by a chain of triangles). In this work it was shown that upon changing the magnitude of the Bjerrum defect formation energy, one may control the proton conductivity for a particular value of proton concentration within the system. It was also shown that for the system constructed by means of triangles, a decrease of the proton conductivity corresponds to a significantly broader range of proton concentrations than is the case for a chain of rods. Furthermore, because of its general definition, the model presented in this work may also be expanded to perovskites, which system could be modeled through a use of a two-dimensional network of squares. It is also important to highlight the fact that despite its undeniable simplicity, the model presented in this work is reliable enough to reproduce experimental data $[8,9]$.

\section{References}

[1] C.H. Lee, H.B. Park, Y.M. Lee, R.D. Lee, Ind. Eng. Chem. Res. 44, 7617 (2005).

[2] A.M. Stephan, T.P. Kumar, M.A. Kulandainathan, N.A. Lakshmi, J. Phys. Chem. B 113, 1963 (2009).

[3] L.Z. Fan, Y.S. Hu, J. Maier, P. Adelheim, P. Smarsly, M. Antonietti, Adv. Funct. Mater. 17, 3083 (2007).

[4] K.-D. Kreuer, Chem. Mater. 8, 610 (1996).

[5] T. Masłowski, A. Drzewiński, J. Ulner, J. Wojtkiewicz, M. Zdanowska-Fracczek, K. Nordlund, A. Kuronen, Phys. Rev. E 90, 012135 (2014).

[6] R.P. Bell, Proc. R. Soc. Lond. A 139, 466 (1933). .

[7] R. Hassan, E.S. Campbell, J. Chem. Phys. 97, 4326. (1992).

[8] T. Masłowski, A. Drzewiński, P. Ławniczak, J. Ulner, Solid State Ion. 272, 166 (2015).

[9] T. Masłowski, A. Drzewiński, P. Ławniczak, M. Zdanowska-Frączek, J. Ulner, Solid State Ion. 278 , 114 (2015). 